\title{
Infección por Serratia marcescens en implante orbitario poroso
}

\section{Infection by Serratia marcescens in porous orbital implant}

\author{
Lorena Fernández-Montalvo ${ }^{*}$, Nuria Ibáñez-Flores², Pilar Cifuentes-Canorea²
}

y Brenda C. Mendoza-García

${ }^{1}$ Servicio de Oftalmología, Hospital Universitario de San Juan de Alicante, Alicante; ${ }^{2}$ Departamento de Órbita y Oculoplastia del Institut Català de la Retina, Barcelona. España

\section{Resumen}

Caso clínico: Se describe el caso de un paciente con antecedentes de evisceración en el ojo izquierdo e implante poroso de polietileno tras un accidente de tráfico tres años antes. Ha presentado exposiciones recurrentes del mismo. En el cultivo de las secreciones conjuntivales se aisló Serratia marcescens, un germen frecuentemente asociado a infecciones nosocomiales, sobre todo en unidades de cuidados intensivos pediátricas, y que es infrecuente en adultos. Discusión: La aparición de secreciones mucopurulentas en presencia de una dehiscencia conjuntival en un paciente con un implante orbitario debe alertarnos de una posible infección profunda del implante. Por ello, deberemos realizar cultivos para detectarla. Cuando el tratamiento antibiótico no puede controlar la infección, se requerirá la explantación del implante.

Palabras clave: Evisceración del ojo. Implantes orbitarios. Complicaciones. Infección. Cavidad anoftálmica. Serratia marcescens.

\section{Abstract}

Case report: We report a case of a patient who underwent left eye evisceration and porous polyethylene implant insertion after a traffic accident 3 years before. He developed recurrent implant exposition. Conjunctival swab culture showed growth of Serratia marcescens, a bacillus associated with nosocomial infections, especially in pediatric intensive care units, but rare in adults. Discussion: Implant infection should be suspected when there is persistent discharge and conjunctival dehiscence after implant insertion. Therefore, all patients with an implant exposure must undergo a culture for infection detection. When even appropriate antibiotics cannot control the infection, the implant must be removed.

Key words: Eye evisceration. Orbital implants. Complications. Infection. Anophthalmic socket. Serratia marcescens.

Fecha de recepción: 23-02-2017

Fecha de aceptación: 21-06-2017 DOI: 10.24875/RMO.M18000024
Disponible en internet: 01-07-2019 Rev Mex Oftalmol. 2018;93(4):200-202 www.rmo.com.mx 


\section{Introducción}

Actualmente los implantes orbitarios de polietileno poroso (PP) son ampliamente utilizados después de una enucleación o evisceración. Las complicaciones reportadas incluyen la aparición de secreciones, dolor persistente, dehiscencia conjuntival, granuloma piógeno, exposición e infección del implante ${ }^{1}$. Aunque esta última es rara, es la complicación más grave debido a que suele conllevar la necesidad de la retirada del implante? ${ }^{2}$.

\section{Caso clínico}

Paciente varón de 48 años eviscerado de urgencia en otro centro, con implante de PP de $20 \mathrm{~mm}$ tras un estallido ocular de su ojo izquierdo secundario a un accidente de tráfico tres años antes. Acude por dolor y molestias con su prótesis desde la cirugía, que no han mejorado a pesar de haberla cambiado en varias ocasiones. En la anamnesis dirigida, el paciente refiere que tras el accidente de tráfico requirió ingreso en la unidad de cuidados intensivos (UCl) durante tres semanas. Al examen oftalmológico se aprecia una dehiscencia conjuntival central con exposición del implante de PP de $6 \times 7$ mm sin signos de infección (Fig. 1).

Ante los hallazgos encontrados al examen físico, se decidió realizar la reconstrucción de la cavidad orbitaria con fresado del implante y colocación de un injerto de pericráneo para cubrir el defecto conjuntival. Se realizó un cultivo de las secreciones que emanaban del implante expuesto, que resultó negativo. La evolución postoperatoria inicial fue lenta pero favorable, con la conjuntivalización completa del injerto en cuatro meses. El paciente siguió controles periódicos hasta que un año después acude a urgencias refiriendo secreciones mucopurulentas y dolor en el ojo izquierdo. En la exploración se observó una dehiscencia conjuntival central con exposición del implante y secreciones blanquecinas (Fig. 2). Se decidió realizar un nuevo cultivo y antibiograma de exudado conjuntival, que resultó positivo para Serratia marcescens. Se pauta ciprofloxacino $750 \mathrm{mg}$ cada $12 \mathrm{~h}$, omeprazol $20 \mathrm{mg}$ cada $24 \mathrm{~h}$ y moxifloxacino hidrocloruro $0.5 \%$ cada $2 \mathrm{~h}$ según antibiograma. Una semana después se realizó el explante del implante y se repitió el cultivo, que confirmó la colonización por S. marcescens. Dado que precisaba un volumen importante, se mantuvo dos semanas sin implante con antibioterapia según antibiograma hasta considerar la infección curada. Tras lo cual presentó una notable mejoría, por lo que se pautó una dosis preoperatoria de ceftazidima intravenosa y se realizó una reconstrucción de la cavidad. Para ello

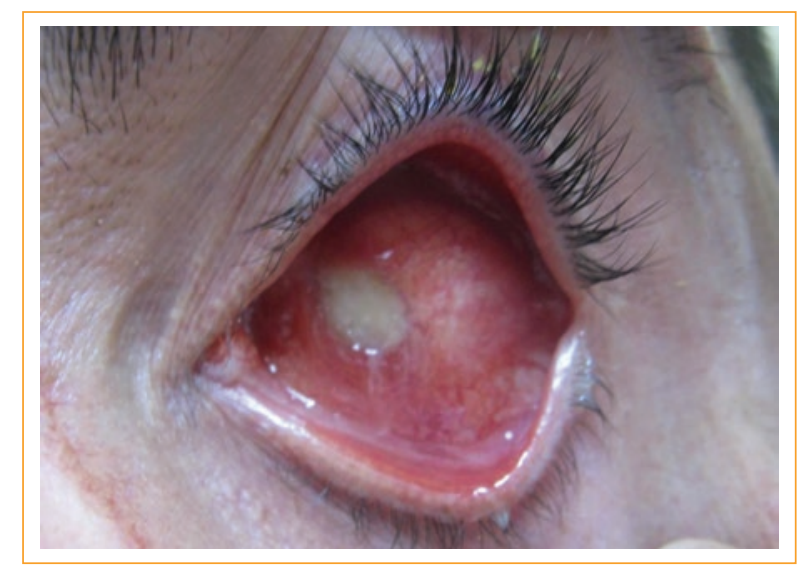

Figura 1. Dehiscencia conjuntival central con exposición del implante de Medpor de $6 \times 7 \mathrm{~mm}$.

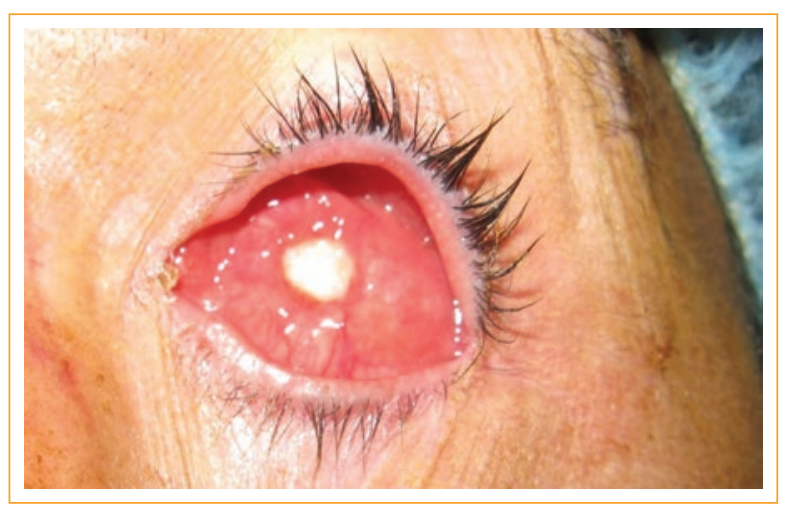

Figura 2. Exposición del implante un año después de la reconstrucción de la cavidad orbitaria con injerto de pericráneo.

utilizamos un implante dermograso de glúteo que se colocó sobre un implante orbitario de PP de $20 \mathrm{~mm}$ con inyección de ceftazidima subconjuntival $100 \mathrm{mg} / 0.5 \mathrm{ml}$ e inyección en tejidos de colirio reforzado de ceftazidima $50 \mathrm{mg} / \mathrm{ml}$. Tras tres años de seguimiento está asintomático, con buen resultado funcional y buena adaptación a la prótesis externa.

\section{Discusión}

Los implantes de PP fueron utilizados como implantes orbitarios por primera vez por Dresner en $1991^{3}$. Se trata de un material sintético (PP de alta densidad), de superficie hidrofóbica y cargada negativamente, que actúa como una capa protectora que inhibe la adherencia de bacterias. Presenta poros de entre 150$400 \mu \mathrm{m}$ de tamaño (media $200 \mu \mathrm{m}$ ) que permiten la integración del tejido orbitario fibrovascular del receptor y la fijación del implante, por lo que disminuye el riesgo de infección y extrusión ${ }^{4}$. 
La complicación más frecuentemente asociada a su uso es la exposición del implante ${ }^{1,5-8}$. La infección del mismo es una complicación rara pero temida por la gravedad que reviste. La puerta de entrada de los gérmenes puede estar en el momento de colocación del implante o postoperatoriamente a través de una zona de dehiscencia conjuntival. Los gérmenes dentro del implante se multiplican y migran a la superficie donde propician la inflamación y dehiscencia de la conjuntiva. Esta puede actuar como puerta de entrada, por lo que el problema se perpetúa. Jung, et al., en una serie de 314 casos con implante orbitario de PP, tuvieron tres casos de infección, sin respuesta al tratamiento médico, en los que se aislaron en el cultivo Staphylococcus aureus en dos casos y Staphylococcus epidermidis en el resto ${ }^{6}$. You, et al, en una serie de pacientes con infección del implante orbitario de hidroxiapatita por $S$. aureus, S. epidermidis, estreptococos $\alpha$-hemolíticos y peptoestreptococos no obtuvieron respuesta al tratamiento médico. El único caso que pudo ser controlado con antibioterapia tenía un implante de $\mathrm{PP}^{8}$. En nuestro caso, el agente causante de la infección del implante resultó ser $S$. marcescens, un bacilo gramnegativo frecuentemente asociado a infecciones nosocomiales graves en $\mathrm{UCl}$, especialmente pediátricas ${ }^{9}$. A pesar de que la endoftalmitis por S. marcescens es rara, ha sido relacionada con blebitis postrabeculectomía, facoemulsificación, queratoplastia penetrante, exposición de cerclaje escleral, exposición de implante de drenaje para glaucoma, queratoprótesis y después de la inserción de un implante orbitario aloplástico tras fractura de suelo orbitario ${ }^{10,11}$. Seyhan, et al. presentan un caso de implante intranasal de PP infectado por S. marcescens siete años después de su inserción en una paciente con enfermedad de Hashimoto que requirió ser explantado ${ }^{12}$. Sin embargo, no hemos encontrado ningún caso publicado de infección de implante orbitario secundario a evisceración. La infección podría haber ocurrido durante el ingreso en la $\mathrm{UCl}$ antes de que la vascularización del implante se desarrollase totalmente (puede llevar varios meses) y los gérmenes quedasen acantonados en el implante en sitios de difícil acceso para los antibióticos y las células defensivas del huésped. Esto explicaría que desde el primer momento tras la evisceración refiriera molestias y un estado de inflamación crónica conjuntival que no mejoraba con tratamiento tópico ni con el recambio de la prótesis.

\section{Conclusión}

La aparición de secreciones mucopurulentas en presencia de una dehiscencia conjuntival en un paciente con un implante orbitario debe alertarnos de una posible infección profunda del implante. Por ello, deberemos realizar cultivos para detectarla. Cuando el tratamiento antibiótico no puede controlar la infección, se requerirá la explantación del implante.

\section{Responsabilidades éticas}

Protección de personas y animales. Los autores declaran que para esta investigación no se han realizado experimentos en seres humanos ni en animales.

Confidencialidad de los datos. Los autores declaran que han seguido los protocolos de su centro de trabajo sobre la publicación de datos de pacientes.

Derecho a la privacidad y consentimiento informado. Los autores han obtenido el consentimiento informado de los pacientes y/o sujetos referidos en el artículo. Este documento obra en poder del autor de correspondencia.

\section{Financiamiento}

Sin financiamiento.

\section{Conflicto de intereses}

Los autores declaran que no existe ningún conflicto de interés de los investigadores en este estudio.

\section{Bibliografía}

1. Jordan DR, Bawazeer A. Experience with 120 synthetic hydroxyapatite implants (FCl3). Ophthal Plast Reconstr Surg. 2001;17:184-90.

2. Jordan DR, Brownstein S, Faraji H. Clinicopathologic analysis of 15 explanted hydroxyapatite implants. Ophtalm Plastic Reconstructive Surg. 2004;20:285-90.

3. Karesh JW, Dresner SC. High-density porous polyethylene (Medpor) as a successful anophthalmic socket implant. Ophthalmology. 1994; 101:1688-95

4. Lee S, Maronian N, Most SP, Whipple ME, McCulloch TM, Stanley RB, et al. Porous high-density polyethylene for orbital reconstruction. Arch Otolaryngol Head Neck Surg. 2005;131:446-50.

5. Lee JH, Yang SW, Choi WV. Clinical outcome and complications of Medpor orbital implant. J Korean Ophthalmol Soc. 2001;42:933-8.

6. Jung SK, Cho WK, Paik JS, Yang SW. Long-term surgical outcomes of porous polyethylene orbital implants: a review of 314 cases. $\mathrm{Br} \mathrm{J}$ Ophthalmol. 2012;96(4):494-8.

7. Alwitry A, West S, King J, Foss AJ, Abercrombie LC. Long-term follow-up of porous polyethylene spherical implants after enucleation and evisceration. Ophthal Plast Reconstr Surg. 2007;23(1)11-5.

8. You Jr, Seo JH, Kim YH, Choi WC. Six cases of bacterial infection in porous orbital implants. Jpn J Ophthalmol. 2003;47(5):512-8.

9. Voelz A, Müller A, Gillen J, Le C, Dresbach T, Engekhart S, et al. Outbreaks of Serratia marcescens in neonatal and pediatric intensive care units: clinical aspects, risk factors and management. Int J Hyg Environm Health. 2010;213:79-87.

10. Equi RA, Green WR. Endogenous Serratia marcescens endophthalmitis with dark hypopyon: a case report and review. Surv Ophthalmol. 2001;46:259-68.

11. Sridhar J, Kuriyan AE, Flynn HW, Smiddy W, Venincasa VD, Miller D. Endophthalmitis caused by Serratia marcescens. Clinica features, antibiotic susceptibilities, and treatment outcomes. Retina. 2015;35:1095-100.

12. Seyhan T, Borman H, Deniz M. Kocer E. Intranasal porous polyethylene implant extrusion 7 years after insertion in a patient with Hashimoto disease. J Craniofac Surg. 2009;20(1):73-4. 